REVISTA DE DERECHO UNED, NÚM. 13, 2013

\title{
CONTRIBUCIÓN A UNA TEORÍA CRÍTICA DE LOS DERECHOS HUMANOS
}

\author{
CONTRIBUTION TO A CRITICAL THEORY OF HUMAN RIGHTS
}

\begin{abstract}
ALÁN ARIAS MARÍN
Catedrático de Filosofía Política en la Facultad de Ciencias Políticas y Sociales de la UNAM; Investigador en el Centro Nacional de Derechos Humanos-CNDH. México

alan.arias@usa.net
\end{abstract}

Resumen: Las consideraciones que se presentan en este artículo se postulan metodológica y heurísticamente a través de tesis, las cuales se inscriben en el horizonte de una contribución a una teoría crítica de los derechos humanos. Las ocho tesis, en su conjunto, expresa temas cruciales para el debate contemporáneo de los derechos humanos, determinado por el debilitamiento de la tradicional hegemonía jurídica y la irrupción pluralista del conjunto de las ciencias sociales. Con ello, se pretende una reconsideración de los derechos humanos bajo premisas críticas y sobre la base de una concepción disímil a la dominante tradición cristiano-liberal.

Abstract: The considerations presented in this article, asume a methodological and heuristic nomination through several theses, which fall within the horizon of a contribution to a critical theory of human rights. The eight theses, as a whole, expresses some decisive themes for the contemporary discussion of human rights, determined by the weakening of the traditional legal hegemony and the emergence of pluralistic set of social sciences. This paper tries a reconsideration of human rights under critical assumptions dissimilar to the liberal Christian tradition. 
Palabras clave: derechos humanos, globalización, multiculturalidad, multidisciplinariedad, víctima.

Keywords: Human rights, globalization, multicultural, multidisciplinary, victim.

Recepción original: 12/11/2013

Aceptación original: 25/11/2013

Sumario: I. Introducción. Premisas conceptuales; II. Tesis 1. Inadecuación entre teoría y práctica; III. Tesis 2. De la globalización y su matriz teórica básica; IV. Tesis 3. Imperativo multicultural; V. Tesis 4. Imperativo multidisciplinario; VI. Tesis 5. Imperativo de género; VII. Tesis 6. Del sufrimiento y la noción de víctima; VIII. Tesis 7. De la construcción de una noción crítica de víctima; IX. Tesis 8. De los derechos humanos y la lucha por el reconocimiento; X. A manera de conclusión.

\section{INTRODUCCIÓN. PREMISAS CONCEPTUALES}

Las tesis que aquí se presentan se inscriben en el horizonte de una contribución a una teoría crítica de los derechos humanos. Es un postulado autocrítico irrenunciable del discurso crítico el riguroso cuestionamiento de las propias posiciones filosóficas, sociológicas y políticas, así como de las relaciones entre ellas; aquí se sostiene que el proyecto y el discurso de los derechos humanos ha de someterse sistemáticamente a tales prácticas auto-correctivas.

La adopción de un perspectiva modulada por la tradición de la teoría crítica supone asumir dos premisas metodológicas fundamentales respecto del concepto derechos humanos. Por un lado, los derechos humanos son considerados como movimiento social, político e intelectual así como (su) teoría propiamente dicha. Su determinación básica, a lo largo de su historia, consiste en su carácter emancipatorio (resistencia al abuso de poder, reivindicación de libertades, regulación garantista por parte del Estado); su sustrato político indeleble la exigencia y afirmación de reconocimiento.

Son simultáneamente proyecto práctico y discurso teórico (lejos de ser sólo derechos). Su consistencia es la de una multiplicidad de prácticas que se despliegan en múltiples dimensiones y se configuran en variados repertorios estratégicos y tácticos ${ }^{1}$; su intencionaldad o

1 Esos serían hipotéticamente los contenidos o notas de los derechos humanos entendidos experimentalmente en tanto que concepto. 
sentido busca la instauración de acontecimientos, acontecimientos políticos, es decir, irrupción de exigencias de reconocimiento que modifican las correlaciones de fuerza y dominio prevalecientes. Esta determinación de su carácter político en sentido estricto (diferenciado de la política), le imprime su sentido instituyente y, en la dimensión propiamente jurídica, como práctica seminal, los derechos humanos como la afirmación del derecho a tener derechos, en la brillante formulación de Hannah Arendt ${ }^{2}$.

De otra parte, los derechos humanos son entendidos como un fenómeno histórico. En tanto que conjunto multidimensional de prácticas y su correspondiente saber e ideología se encuentra especificado históricamente; los factores históricos y las condiciones sociales, políticas y culturales conforman variables indispensables para comprender y explicar su desarrollo previo y su caracterización actual.

El presente ensayo, construido mediante la formulación de tesis busca, amén de acentuar con esa modalidad su intencionalidad heurística, comulgar con las formas precursoras e iniciales -fragmentos y no sistemas- de montaje o collage típicas del discurso de la teoría crítica ${ }^{3}$. Con estos modos se pretende una reverberación del hecho de que, si bien ha habido importantes intervenciones críticas en el trayecto histórico de los derechos humanos, no se ha propuesto una reconsideración de ese proyecto humanístico bajo explícitas premisas críticas y, mucho menos, articulada con los presupuestos teóricos de una concepción radicalmente disímil a la tradición cristiano-liberal de los derechos humanos (al menos de 1948 a la fecha).

\section{TESIS 1. INADECUACIÓN ENTRE TEORÍA Y PRÁCTICA}

La exigencia contemporánea de una aproximación crítica a los derechos humanos se justifica, en primera instancia, por la no correspondencia entre el desarrollo discursivo y normativo del proyecto de los derechos humanos y su situación práctica de crecientes vulneración, irrespeto y manipulación de los mismos. Así como también, en segunda instancia, en virtud de la percepción y el diagnóstico respecto a su

2 ARENDT, H., Sobre los orígenes del totalitarismo, Madrid, Alianza, 1982, pp. 379-382.

3 La denominada primera generación de la Escuela de Frankfurt, Max Horkheimer, Theodor W. Adorno, Herbert Marcuse, Erich Fromm y, un tanto excéntricamente, Walter Benjamin. 
situación de crisis teórica, crisis conceptual y cultural presente en sus dimensiones tanto externa como interna.

En lo exterior, expresada en la paradoja de ser -hoy por hoy- un discurso referencial dominante, en términos valorativos y normativos y, al mismo tiempo, ser objeto de instrumentalizaciones políticas, manipulaciones legitimatorias, así como de un uso banal y un abuso vulgarizador del lenguaje de los derechos humanos, por un lado. Y, por otro lado, en su dimensión interna propiamente discursiva, en cuanto a la radical inadecuación de su composición conceptual y sus proposiciones teóricas respecto de las efectivas condiciones sociales, políticas y culturales del momento histórico contemporáneo. La consecuencia indeseada y/o perversa es la pérdida de sus potencialidades emancipatorias.

El discurso actual dominante de los derechos humanos -su formulación hegemónica juridicista- no es expresión teórica suficiente de las necesidades prácticas del proyecto-movimiento de los derechos humanos en las condiciones actuales, tanto en sus medios e intrumentos como en sus objetivos. Existe, desde hace décadas, la imposibilidad de vincular directa y adecuadamente la práctica y la teoría de los derechos humanos a la forma original renovada correspondiente a su refundación contemporánea.

La figura histórica de los derechos humanos, en su fase de reformulación y desarrollo, surgió reactivamente luego del final de la Segunda Guerra Mundial. Ese discurso, matriz normativa y teórica de toda la evolución posterior -su forma «clásica»- no fue expresión adecuada de las nuevas condiciones emergentes del mundo de la posguerra, ni contó con un diagnóstico, acorde a sus propias finalidades, respecto de las tensiones de la llamada Guerra Fría, que caracterizaron a la segunda mitad del siglo XX, prácticamente hasta los años 90. Mucho menos ha sido capaz de captar y representar de modo teóricamente pertinente y prácticamente viable el desarrollo posterior al colapso del socialismo real, así como las determinaciones del proceso de globalización con una interpretación de la matriz teóricoconceptual derivada de ella. Resultado de esos déficits conceptuales y culturales, el discurso y el movimiento de los derechos humanos vive una crisis práctica y teórica que reclama un replanteamiento crítico y, consecuentemente, un argumento re-legitimador.

En rigor, los derechos humanos en su formulación actual dominante, no son sino el resultado sintético de la situación dramática precedente, con la emergencia de la barbarie absoluta en los campos de exterminio, aludida con el concepto-paradigma Auschwitz; se tra- 
tó de una reacción ilustrada, de rescate de valores y principios éticos de matriz liberal-cristiana. Sin embargo, el optimismo respecto de un posible regreso a valores de convivencia civilizada, normada por el derecho, sobre la base de la dignidad humana, no apreciaba en toda su radicalidad el golpe devastador infligido a toda pretensión teórica y política del proyecto mismo de la Ilustración.

Lo anterior ayuda a entender, si bien parcialmente, por qué es que las propuestas teóricas de los derechos humanos y sus traducciones jurídicas positivas, resultan asequibles y útiles (aún si en un plano de mera denuncia) en condiciones particulares de crisis humanitarias y durante periodos delimitados, en ambientes represivos nugatorios de los derechos civiles y políticos, propios de dictaduras y/o Estados autoritarios; pero resultan inaplicables, inviables, en términos generales y en las condiciones mayoritariamente predominantes en Estados con regímenes razonablemente democráticos.

Las potencialidades de un desarrollo vivo, creativo, del proyecto y el discurso de los derechos humanos ha resultado obstaculizado por las modificadas condiciones históricas de las sociedades y los Estados a través de la segunda mitad del siglo XX y lo que va del presente. Es por ello, pertinente y adecuado un replanteamiento crítico que tome en consideración los factores históricos y asuma con radicalidad las condiciones sociales, políticas y culturales actuales para ensayar una reformulación (una re-legitimación) contemporánea de los derechos humanos.

\section{TESIS 2. DE LA GLOBALIZACIÓN Y SU MATRIZ TEÓRICA BÁSICA}

La complejidad inherente al debate contemporáneo de los derechos humanos encuentra ciertas claves de comprensión si se le relaciona con las condiciones de su especificación histórica. Los grandes cambios sociales, políticos y económicos del siglo XX están determinados por el proceso de globalización, la especificidad contemporánea encuentra su configuración principal en la globalización. No obstante, la conexión entre el discurso de los derechos humanos y el proceso globalizador aparece mediado por una matriz teórica básica; dotada de principios constructivos y operacionales prácticomateriales y también conceptual-culturales generados por las condiciones inherentes de la globalización, sus tendencias determinantes y sus tensiones polarizantes. 
Las condiciones actuales de la sociedad globalizada muestran, por un lado, una fuerte tendencia hacia la homogeneización, posibilitada por pautas económicas y culturales -estándares, hábitos y modas a partir del consumo- extendidas por todo el mundo; y, no obstante, por el otro lado, el reforzamiento de una heterogeneidad cultural a partir de la reivindicación de identidades étnicas, religiosas, culturales y hasta de modos de vida de diverso tipo, que determinan que -en dichas condiciones sociales y culturales- unas y otras cohabiten en el seno de una tensa paradoja.

Un discurso renovado de los derechos humanos podría afirmarse como un territorio discursivo de mediación -y no sólo referencial normativo- entre la afirmación de los universales, con su cuota correspondiente de violencia (universales impuros), de matriz occidental y el cuestionamiento radical de los relativismos culturales y los particularismos nacionales, étnicos, religiosos y lingüísticos (el desafío multicultural a Occidente).

Paradoja de bipolaridad persistente, que no tiende a resolverse a favor de uno de los polos en tensión -homogeneización o heterogeneidad- sino que, más bien, genera un campo de fuerzas de complejas tensiones, pues a medida que las relaciones sociales se amplían, se produce también una intensificación de las diferencias, lo que indica que los procesos globalizadores carecen de esa unidad de efectos que generalmente se da por sentada al hablar de globalización.

Así, el término globalización se suele relacionar con la aprehensión de su carácter irresuelto, sus tensiones contradictorias y sus efectos indeseados: de la "sociedad de riesgo» (Beck) o "sociedad líquida» (Bauman), con espacios que fluyen (Castells), (en) un «mundo turbulento» (Rousenau) y «desbocado» (Giddens), susceptible al "choque de civilizaciones» (Huntington) fundamentado a partir del surgimiento de un «sistema mundial capitalista» (Wallerstein) y que produce, como efecto de su carácter paradojal, procesos de «individualización»(Beck), «retribalización»(Maffesoli), «transculturalización» y «reterritorialización»(García Canclini) ${ }^{4}$.

4 Véase, BECK, U., La sociedad del riesgo mundial: en busca de la seguridad perdida. Barcelona, Paidós, 2008; BAUMAN, Z., Tiempos líquidos, Barcelona, Tusquets, 2007; CASTELLS, M., La era de la información, tomo I, México, Siglo XXI, 2002; ROUSSENAU, J., Distant Proximities: Dynamics Beyond Globalization, Princenton, Princenton University Press, 2002; GIDDENS, A., Un mundo desbocado, Madrid, Taurus, 1999; WALLERSTEIN, I., El moderno sistema mundial II. El mercantilismo y la consolidación de la economía-mundo europea, 1600-1750, México, Siglo XXI, 1998; BECK, U. y GERNSHEIM, E., La individualización: el individualismo institucionalizado y sus consecuencias sociales y políticas, Barcelona, Paidós, 2003; MAFFESOLI, 
Así, escuetamente entendido, podemos señalar que la globalización es un fenómeno social emergente, un proceso en construcción, una dialéctica dotada con sentidos contrapuestos, opciones de valor ineludibles, con carga ideológico-política y de matriz económico-tecnológica. La globalización, bajo la determinación de su fuerte variable económica, forma parte del viejo proceso -siempre creciente- de mundialización del sistema capitalista (teorizado de modo canónico por Marx $)^{5}$. Se trata de una fase de peculiar intensidad del sentido expansivo de la valorización del capital, desdibujando las distinciones clásicas entre mercado local y mundial, ciudad y campo y entre trabajo manual e intelectual (trabajo productivo e improductivo). Esta fase está cargada de implicaciones sociales y culturales condicionadas desde una novedosa y revolucionaria base informática y cibernética, características de la época contemporánea, que problematizan los códigos de la producción de verdades y que realizan rotundamente la tendencia de que las fuerzas productivas principales, las que más y mejor valorizan valor, sean la ciencia y la técnica.

\section{TESIS 3. IMPERATIVO MULTICULTURAL}

La globalización también ha alterado el significado contemporáneo de la soberanía política y jurídica y, con ello, se ha agudizado un debilitamiento de las estructuras estatales frente al escenario global. El desplazamiento de la centralidad del Estado (y su soberanía) se contraponen y colisionan, determinando espacios y tiempos de incertidumbre, agravados por nuevos tipos de violencia (algunos extremos como la violencia del terrorismo y el narcotráfico en algunos países) donde, con la participación del Estado, los derechos humanos quedan situados en una tensa ambigüedad crítica.

Con el fin del bipolarismo global, un conjunto de fuerzas, reacciones, viejas reivindicaciones y aspiraciones encontraron en la afirmación de la heterogeneidad un punto focal; se constituyó, así, en el motor del principio de autonomía y en el potencial constructo de las identidades individuales y colectivas. El poderoso imperativo multicultural -especie de gran paraguas teórico y cultural de las diferencias- se convierte en un desafío e impele a un diálogo con las culturas periféricas, pero también en el seno mismo de las socieda-

M., El tiempo de las tribus, México, Siglo XXI, 2004; GARCÍA CANCLINI, N., La globalización imaginada, Barcelona, Paidós, 1999.

5 MARX, K., El capital (8 volúmenes), Vol. 1, México, Siglo XXI, 1977, pp. 179-214. 
des democráticas de Occidente, respecto a las reivindicaciones valorativas de diferencia y reconocimiento culturales.

Esta irrupción del pluralismo y la heterogeneidad en disputa con el universalismo y la homogeneidad, todavía dominantes -aunque erosionados-, se encuentra indisolublemente asociada a la figura del Estado. La tensión entre derechos humanos, (cuyo horizonte intelectual y derechos positivizados se ubican tradicionalmente en un plano de adscripción universal y bajo un principio de igualdad general) y, el multiculturalismo (como reconocimiento a las diferencias de pertenencia cultural e identidad particulares), surge cuando las demandas de grupos culturalmente diferenciados (reticentes a la aceptación del significado universalmente válido de los valores y las finalidades paradigmáticamente expresados en la forma democrática y en los derechos humanos), resultan imposibles de reivindicar -inasimilables- sin desprenderse de su interrelación con el Estado, ese espacio político -de supuesta igualdad universal- integrado a partir de conceptos universales y presuntas condiciones de homogeneidad.

No obstante, lo que prevalece es la confrontación práctica e intelectual, toda vez el carácter inescapable del conflicto de valores implícito en el impulso históricamente dominante de la perspectiva Occidental y sus formas político culturales (derechos humanos incluidos). Así, las contradicciones se precipitan al territorio dirimente de la política y la lucha por el reconocimiento como condición básica de la construcción y entendimiento de los derechos humanos.

En esa discusión, la temática de los derechos humanos ha ocupado un lugar central, tanto como objeto de crítica valorativa, toda vez que su construcción y fundamentación se han realizado en clave monocultural (occidental), así como por el desarrollo de un debate de revaloración, redefinición y relegitimación del discurso y la teoría de los derechos humanos de cara a las modificadas condiciones de nuestras sociedades globales.

\section{TESIS 4. IMPERATIVO MULTIDISCIPLINARIO}

Derivada de la matriz teórica básica generada por la globalización, sus consecuencias y determinaciones, en particular una de ellas, la del debilitamiento crítico del Estado nacional y de la noción dura de soberanía, es que operan condicionando el movimiento y la teoría de los derechos humanos, por ello, se ha inducido recientemente una mutación en el discurso juiridicista dominante. Un des- 
ajuste crítico que tiende a desplazar al derecho del centro dominante en el discurso de los derechos humanos y que propicia la irrupción del conjunto de las ciencias sociales y la filosofía en su conformación y desarrollo internos.

El impacto de este desarrollo crítico de la teoría de los derechos humanos no ha sido en referencia exclusiva del ámbito jurídico, sino que se ha extendido al de las ciencias sociales en su conjunto; ha inducido una relativización de sus respectivos campos de conocimiento y una interrelación más intensa entre las distintas disciplinas; asimismo, en ciertos territorios, como la filosofía del derecho y la filosofía política, a un radical y complementario intercambio conceptual. De lo que se desprende un imperativo multidisciplinario al discurso de los derechos humanos; exigencia que interpela toda pretensión crítica y de adecuación a las circunstancias reales de una teoría actualizada de los derechos humanos. La complejización, extensión y debilitamiento del derecho como la modalidad hegemónica en la descripción, constitución y legitimación teorética de los derechos humanos ha conducido a la necesidad de una aproximación multidisciplinaria.

El movimiento y el discurso de los derechos humanos son tema relevante y esencial, referente obligado tanto política como jurídica y socialmente, en el debate contemporáneo. La complejidad y riqueza que engloba el concepto derechos humanos, nos impele a trasladar su estudio -migración cultural- hacia una perspectiva más amplia que la generada por la especialización actual de las disciplinas del conocimiento humano. Si bien es cierto que el estudio del tema nos ha remitido, tradicionalmente, al terreno jurídico, también es cierto que el debate y la investigación están lejos de agotarse en ese ámbito. El otrora discurso dominante del Derecho se ha visto impelido a un replanteamiento radical respecto de los derechos humanos y a enfrentar inéditos problemas conceptuales, así como numerosos desafíos teóricos y metodológicos en ese ámbito.

\section{TESIS 5. IMPERATIVO DE GÉNERO}

El feminismo y los estudios de género tuvieron un desenvolvimiento intelectual y un arraigo material inusitado y exitoso a lo largo del siglo pasado. Si alguna revolución cultural contemporánea se mantiene invicta esa es la del feminismo contemporáneo (con todo y sus contradicciones, divisiones y diásporas). Al igual que otros movimientos sociales radicales que reivindican reconocimiento, insertan 
la cuestión propia de las diferencias dentro del lenguaje universalista de los derechos humanos. Propiamente, el discurso feminista es uno que emplaza el debate sobre los derechos humanos a partir de la subversión de la distinción entre universalidad y diferencia.

La coincidencia epocal en el surgimiento tanto del pensamiento político liberal de la Declaración de los Derechos del Hombre y del Ciudadano como del pensamiento feminista emergente, ambos a finales del siglo XVIII, ha inducido, al menos, dos principios definitorios de la disociación entre feminismo y derechos humanos; por un lado, respecto del universalismo de las Declaraciones canónicas y, por otro lado, la afirmación de presupuestos implícitos en la perspectiva del concepto de género, la noción de diferencia y de los recursos conceptuales y políticos, presentes en la tradición feminista.

Género es un (relativamente) nuevo concepto, que además de su inherente ánimo crítico, contiene pretensiones políticas reivindicativas radicales. Con esto, no se trata sólo de situar la noción de género en relación con la perspectiva interpretativa que lo tiene como matriz, esto es, con los movimientos feministas, sino la de enfatizar su carácter esencialmente político.

El concepto de género es simbolización de la diferencia sexual; aquí lo propiamente simbólico consiste en la institución de códigos culturales que, mediante prescripciones fundamentales -como es el caso de las de género- reglamentan el conjunto de la existencia humana en sociedades y periodos históricos específicos ${ }^{6}$. Esta simbolización cultural de la diferencia anatómica sexual toma forma en un conglomerado de prácticas, ideas, discursos y representaciones sociales que influyen y condicionan la conducta objetiva y subjetiva de las personas en función de su sexo.

La noción de género ofrece la posibilidad de pensar el carácter de constructo cultural que tienen las diferencias sexuales, el género es una producción social y cultural históricamente especificada, más allá de la propia estructuración biológica de los sexos, de las identidades de género, de su función y relevancia en las organizaciones sociales. Desde luego, es relevante el papel innegable y paradigmático que opera en la estructuración de la igualdad y la desigualdad en las sociedades.

6 LAMAS, M. (comp.), El género. La construcción cultural de la diferencia sexual, México, Programa Universitario de Estudios de Género-UNAM/Miguel Ángel Porrúa, 1996. 
Asimismo, detrás de los movimientos reivindicatorios, y en particular del movimiento feminista, existe una «semiotización de lo social ${ }^{7}$; esto es, que la fuerza inventiva del movimiento feminista, su contribución, no sólo pasa por las posibilidades heurísticas del concepto y la perspectiva de género sino también por todo lo que deriva de su potencial crítico y desconstructor de ciertos paradigmas teóricos, pero también prácticos ${ }^{8}$. Con ello, tal semiotización de lo social debe entenderse como el sello del horizonte epistemológico contemporáneo; resultado de las estrategias teóricas más diversas, desde la recuperación de la dimensión del sentido de historicistas y hermeneutas, hasta el giro lingüístico de estructuralistas, postestructuralistas y filósofos del lenguaje, y que el arribo conclusivo a tesis establecidas y ya referenciales teóricamente como la de que «toda relación social se estructura simbólicamente y todo orden simbólico se estructura discursivamente» ${ }^{9}$.

Los afanes teóricos del feminismo no son fáciles de deslindar de la política feminista. Con su práctica política las feministas contribuyeron a cimbrar ciertos paradigmas de la derecha y de la izquierda acerca de cómo pensar y hacer política. La posición teórica feminista emplazó, a través de la idea de género, la desarticulación de ciertos paradigmas de la modernidad y de la lógica esencialista en que se sustentan.

Dos de los principales dispositivos teóricos criticados, en su momento, por la teoría feminista, el cuestionamiento del paradigma liberal y sus ejes fundamentales, el racionalismo y el humanismo, inciden directamente en el corpus conceptual de la configuración clásica dominante del discurso de los derechos humanos. La hostilidad histórico-emblemática del feminismo respecto de las teorías embrionarias de derechos humanos (plasmada en la condena a la guillotina de la «girondina» Olympe de Gougés, opuesta a la ejecución del rey y autora de la malhadada Declaración de los derechos de la mujer y de la ciudadana...), encontró respaldo teórico y conceptual sólido (aunque tardío), mediante la problematización con perspectiva de género, de la desigualdad y la discriminación de las mujeres en las concepciones, textos y prácticas originarios del movimiento y el discurso de los derechos humanos.

7 GUTIÉRREZ, G., «El concepto de género: una perspectiva para pensar la política», en La ventana, n. ${ }^{\circ}$ 5, México, Universidad de Guadalajara, 1997, p. 59.

8 GUTIÉRREZ, G., op. cit., pp. 60 y sigs.

$9 \quad$ Ibid, p. 59. 
El arraigo de las teorías feministas en los modos culturales y de pensamiento contemporáneos, su distancia crítica respecto al proyecto y discurso de los derechos humanos, ha mostrado desconstructivamente las inconsistencias de su matriz universalista; asimismo, ha cuestionado el prejuicio radical de la izquierda, especialmente la de corte marxista, que no permitía incorporar y reconocer un sus organizaciones y en su discurso la especificidad de la problemática de género, de su origen y carácter propiamente cultural y que -con ellonegaba e invisibilizaba la marginación, el menosprecio y la subordinación de las mujeres en el universo político cultural de la izquierda.

\section{TESIS 6. DEL SUFRIMIENTO Y LA NOCIÓN DE VÍCTIMA.}

Una de las cuestiones trascendentes que el discurso crítico de los derechos humanos no puede soslayar, es la pregunta acerca si la teoría social y filosófica del siglo XXI será capaz de encontrar significado al sufrimiento humano socialmente generado. La validez y autenticidad del empeño crítico del discurso de los derechos humanos ante el sufrimiento de las víctimas, sólo podrá ser reivindicado y sustentado si mantiene la consciencia alertada respecto del reconocimiento de la fragilidad de las pretensiones de la teoría crítica, así como de la condición malamente existente de los derechos humanos en la actualidad.

El discurso crítico de los derechos humanos, en tanto que saber práctico alimentado de prácticas de resistencia, tiene que ser parte activa en esta des-construcción de los relatos socio-estatales de integración y consuelo del sufrimiento. La militancia de los derechos humanos al lado de las víctimas y el compromiso de su teoría con el desentrañamiento crítico de lo que provoca el sufrimiento, la violencia y la vulneración de la dignidad de las personas, impone nuevas tareas a la agenda teórica y práctica del movimiento de los derechos humanos.

La meditación acerca del sufrimiento resulta inexcusable en la actualidad, en tanto que aparece como la vía material que comunica tanto con la noción de víctima así como con el concepto de dignidad. Para la teoría contemporánea de los derechos humanos, la relación entre violencia y dignidad vulnerada no es directa. Está mediada por la (noción) de víctima. Tanto la violencia como la dignidad humana (vulnerada) son perceptibles a partir de la vida dañada en las víctimas, cuyo registro radica en las narrativas del sufrimiento. 
Una perspectiva crítica de la idea de víctima propicia la apertura a una doble dimensión epistemológica, tanto propiamente cognoscitiva como en su función heurística: a) la víctima es punto de partida metodológico, plausible para una investigación crítica del núcleo básico ético de una teoría de los derechos humanos, a partir del estudio de la violencia; b) la víctima es la mediación necesaria con la dignidad dañada o vulnerada que se implica en ella, toda vez que la aproximación o el asedio conceptual a la idea de dignidad humana sólo ocurre idóneamente por vía negativa, esto es, a través de las múltiples formas de daño y de vulneración de la dignidad de las personas.

La revisión crítica de la noción de víctima, de alta complejidad y riqueza de determinaciones, supone asumirla como la mediación plausible entre las nuevas determinaciones y modalidades de la violencia estatal y societal contemporánea respecto de la dimensión de la dignidad humana.

El apelar a las violaciones de la dignidad humana en el siglo XX, con el involucramiento del discurso de los derechos humanos en ello, posibilitó el descubrimiento de la función heurística de la noción de víctima y, con ello, el concepto de dignidad humana pudo cumplimentar con su tarea como fuente de ampliación de nuevos derechos.

No obstante, resulta pertinente desconstruir críticamente la noción de la dignidad humana, asumirlo como una noción vacía de contenidos conceptuales y/o como derivada de alguna fundamentación axiomática particular (de imposibles consensos); apelar a un uso del concepto de dignidad como postulado de la razón práctica contemporánea, como referente de potencialidad normativa para la convivencia social ${ }^{10}$. La dignidad humana vulnerada por la violencia tiende a convertirse, entonces, en la vía que constata y confirma, en clave de derechos humanos, la condición de víctimas, en el criterio que pondera y reconoce su sufrimiento y el horizonte proyectivo de su emancipación.

La revisión crítica de la noción de víctima, con la mira en la pretensión de contribuir a una fundamentación ética de los derechos humanos, supone asumirla como la mediación plausible entre las nuevas determinaciones y modalidades de la violencia estatal y societal contemporáneas con la dimensión de la dignidad humana. ${ }^{11} \mathrm{Su}$ estudio, resulta un asunto crucial para el discurso social, filosófico y

10 Para un desarrollo más extenso de esta idea ver, ARIAS MARÍN, A., «Derechos humanos: entre la violencia y la dignidad», Revista Derechos Humanos-México, CNDH, n. ${ }^{\circ}$ 19, México, 2012, pp. 33-34.

11 Ver ARIAS MARÍN, A., op. cit. 
jurídico de los derechos humanos. Análisis y reinterpretación de la ecuación discursiva señera de los derechos humanos, el clásico nudo fundamental -históricamente siempre repensado- de la relación violencia-víctima-dignidad. Como se sabe, la relación entre violencia y dignidad vulnerada no es directa, se encuentra mediada por la noción de víctima, de ahí su importancia teórica y metodológica. Así, la problemática generada por el tratamiento crítico $^{12}$ de esos temas constituye actualmente -como desde su origen- la columna vertebral de los derechos humanos.

\section{TESIS 7. DE LA CONSTRUCCIÓN DE UNA NOCIÓN CRÍTICA DE VÍCTIMA}

La perspectiva crítica de la idea de víctima -como vimos en la tesis anterior- propicia la apertura a una doble dimensión epistemológica, tanto propiamente cognoscitiva como en su función heurística: a) la víctima es punto de partida metodológico, plausible para una investigación crítica del núcleo básico ético de una teoría de los derechos humanos, a partir del estudio de la violencia; $b$ ) la víctima es la mediación necesaria con la dignidad dañada o vulnerada que se implica en ella, toda vez que la aproximación o el asedio conceptual a la idea de dignidad humana sólo ocurre idóneamente por vía negativa, esto es, a través de las múltiples formas de daño y de vulneración de la dignidad de las personas, expresadas en las narrativas del sufrimiento.

Ahora bien, la noción de víctima, en la evolución y cristalizaciones de sus significados, es una noción vaga, cargada de polivalencia semántica y de polisemia cultural, donde los significados sacrificiales resultan dominantes. De entrada, estimula aproximaciones intuitivas y favorece los prejuicios, fuentes principales de los obstáculos epistemológicos al conocimiento. El modo de trabajo o procesamiento racional sobre el concepto de víctima ha tenido tradicionalmente la deriva dominante del derecho, de manera que la noción de víctima con mayor y mejor carga intelectual resulta ser predominante y unidimensionalmente jurídica. La parafernalia técnico-administrativa relativa al interés pragmático, propio del saber jurídico, ha resultado ser velo y complemento de los significados de sacrificio y resignación inherentes a la idea de víctima, contenidos arcaizantes y de corte teológico.

\footnotetext{
12 Véase este argumento con mayor detalle en ARIAS MARÍN, A., op. cit., pp. 16
} y sigs. 
La crítica reflexiva y práctica respecto al concepto de víctima lleva a un replanteamiento respecto de ideas y prácticas asociadas con ella. Indefensión, sometimiento, debilidad, reconocimiento negativo como meras víctimas, al final, predominio de variadas formas de menosprecio, redundan en un bajo de potencial de protesta, una restricción de sus alcances organizativos, convocatorias de solidaridad compasiva, manipulaciones políticas y facilidades al chantaje de las víctimas indirectas: la noción convencional de víctima se limita al umbral de la queja victimante y no alcanza la proclama de la protesta, ni logra acceder a la conformación de un discurso teórico y práctico crítico y transformador de su condición yaciente, adolorida y subordinada ${ }^{13}$.

Amén de todos esos elementos, que son intrínsecos, inmanentes, al concepto de víctima, hay que considerar los factores extrínsecos, trascendentes, tales como: el exceso de violencia y su correlativo plus de sufrimiento socialmente producido, así como la consecuencia de una multiplicación de potenciales víctimas en las actuales circunstancias de las sociedades de riesgo contemporáneas. Estos factores extrínsecos, que configuran el entorno o contexto que induce (potencia o estimula) un exceso de sufrimiento social inasimilable, inducen perentoriamente la necesidad de procurar un concepto de víctima complejo, amplio, dinámico y funcional para lidiar mejor (procesar adecuadamente) esa sobrecarga de violencia sobre la sociedad.

\section{TESIS 8. DE LOS DERECHOS HUMANOS Y LA LUCHA POR EL RECONOCIMIENTO}

Es cierto que se hace y se puede hacer política con los derechos humanos, se les puede instrumentalizar y utilizar para objetivos ajenos, políticamente correctos o impresentables, al servicio de los de arriba o los de abajo, por el mantenimiento del statu quo o su modificación, igualitarios o para agudizar las desigualdades. No obstante, esas instrumentalizaciones políticas, no eliminan el sentido político inmanente propio de los derechos humanos.

Ese sustrato, lo intrísecamente político del proyecto y el discurso de los derechos humanos, radica en que lo específico y común de esas múltiples prácticas de resistencia, reclamo, imposición y emplazamiento de actos, hechos o acontecimientos de tensión de la corre-

13 Algunas de las premisas para la construcción de un concepto crítico de víctima son revisadas en ARIAS MARÍN, A., «Aproximación a un concepto crítico de víctima en derechos humanos", Revista Derechos Humanos México, n. ${ }^{\circ}$ 20, CNDH, 2012. 
lación de fuerzas conlleva e implica exigencias de reconocimiento. Ese carácter consiste en emplazar relaciones de poder en términos de reconocimiento, de lucha por el reconocimiento, en otras palabras, instaurar acontecimientos políticos, tal es lo que define lo esencialmente político de los derechos humanos ${ }^{14}$.

Al resistir, al decir: «¡no!, ¡basta!, ¡así no!, ¡no más! Los individuos y grupos se oponen al abuso de poder, pero también reivindican, emplazan, estatuyen una exigencia de reconocimiento respecto del otro; el que violenta, abusa, explota...¿¿Reconocimiento de qué?; de la dignidad, responde el discurso de los derechos humanos, de la alteridad en pie de igualdad en virtud de ser sujetos libres. Reivindican emancipación, libertades -derechos-, regulaciones, garantías (de cara al Estado); se plantan libremente, en pie de igualdad; con dignidad, se dice. Dignidad que es discernible, constatable y afirmable sólo por vía negativa: ante su denegación, el abuso y las violencias que vulneran la dignidad; esto es, ante la negación de las libertades y la desigualación de los iguales (la materialización de los procesos de exclusión, estudiados ejemplarmente por Foucault y el proceso de la desigualdad, modélicamente analizado por $\operatorname{Marx}^{15}$ ).

Desde esa radicalidad, relativa al carácter político intrínseco de los derechos humanos, es que resulta adecuado suponer que estarían en condiciones de posibilidad de sobreponerse al politicismo inherente en la calificación de las víctimas y la autodesignación de las mismas y -asimismo- coadyuvar al diseño e instrumentación de una política, entendida como disciplina ante las consecuencias del acontecimiento, una política victimológica en clave de derechos humanos.

La noción crítica de víctima, en tanto que elemento apto para coadyuvar a una fundamentación ética de los derechos humanos, asume un papel trascendente en la lucha de sujetos que reivindican aspectos no reconocidos de su identidad -por la vía de la conciencia de haber sufrido una injusticia. Es a partir de este momento que la víctima, al igual que los maltratados, excluidos o despreciados, diversos grupos victimizados que han experimentado formas de ne-

14 Se haría necesario para el argumento la pertinencia de la distinción entre lo político y la política; no es aquí el momento (ni hay el espacio) para tal desarrollo. En ese sentido ver: LEFORT C., La incertidumbre democrática. Ensayos sobre lo político, Barcelona, Anthropos, 2004; y MOUFFE, C., El retorno de lo político, Barcelona, Paidós, 1999.

15 Para una presentación sintética de esos procesos en contexto de derechos humanos, ver ARIAS MARÍN, A., "Globalización y debate multicultural. Un nuevo imperativo contemporáneo», Revista Derechos Humanos México, México, n. ${ }^{\circ}$ 12, 2008. 
gación del reconocimiento, no sólo sufren a partir del menosprecio de su condición, sino que, pueden descubrir que el menosprecio en sí mismo puede generar sentimientos, emociones y el impulso moral que motivan e impelen comportamientos y acciones (prácticas) para devenir en sujetos activos de luchas por reconocimiento.

Situar el concepto de reconocimiento, con su potencial carácter crítico, des-constructivo, en la construcción de un concepto crítico de víctima (complejo, abierto, dinámico, funcional), significa asumir la centralidad del conflicto bajo un entendimiento de su función positiva (creativa) de integración social, a condición de que se le deje de ver de un modo limitado y negativo, como ha sido el caso desde la perspectiva teórica dominante. Las luchas de reconocimiento, históricamente, han generado la institucionalización de ciertas prácticas sociales que evidencian el pasaje de un estadio moral a otro más avanzado -un aumento de la sensibilidad moral, señala Honneth ${ }^{16}$.

La lucha de los grupos sociales por alcanzar formas cada vez más amplias de reconocimiento social se convierte, muta, en una fuerza estructurante del desarrollo moral de la sociedad. Esa ha sido el sentido humanista del movimiento y la teoría de los derechos humanos; toca a su reformulación crítica, insistir en la articulación de la noción -yaciente- de víctima con un proyecto -enhiesto- de resistencia y emancipación.

Así, en dicha perspectiva, la lucha social no puede explicarse sólo como resultado de una lucha entre intereses materiales en oposición sino también como consecuencia de los sentimientos morales de injusticia; una gramática moral de los conflictos sociales ${ }^{17}$. La víctima, cuya visibilidad es posible a través del sufrimiento, se constituye primordialmente en esa imagen inicial de injusticia; no debiera permanecer en la queja sino levantarse para la proclama (los derechos humanos pudieran ser el revulsivo de esa metamorfosis).

\section{A MANERA DE CONCLUSIÓN}

No mantenerse en la insistencia de lo negativo -de la crítica-, pasar demasiado rápidamente a la afirmación de lo positivo, favorece en realidad la perpetuación de lo existente falso, de lo malamente existente, más que servir a su superación.

16 HONNETH, A., Reconocimiento y menosprecio. Sobre la fundamentación normativa de una teoría social, Buenos Aires, Katz editores, 2010, p. 37.

17 HONNETH, A., The Struggle for Recognition. The Moral Grammar of Social Conflicts, Cambridge, The MIT Press, 1995. 
El conjunto de las tesis que se han presentado constituyen un esbozo inicial de exploración, las cuestiones están dispuestas y orientadas a un trabajo mayor, más acabado, y, por ende, construido para una posterior conformación. No obstante, apuntan horizontes de indagación al igual que la tensión crítica atisba núcleos problemáticos que reclaman una reactivación del pensamiento crítico, expectativa vigente en el discurso y cultura de los derechos humanos. 